\title{
Thyroid hormone levels and mortality after heart transplantation
}

A. Nagy1 , E. Holndonner-Kirst², C. Eke 3 , B. Baukó3 ${ }^{3}$ J. Gál2, A. Szekely² Semmelweis University, School of PhD studies - Budapest (Hungary), ${ }^{2}$ Semmelweis University, Department of Anaesthesiology and Intensive Therapy - Budapest (Hungary), ${ }^{3}$ Semmelweis University, Faculty of MedicineBudapest (Hungary)

\section{Background and Goal of Study:}

Thyroid hormone levels decreases after acute, serious systemic stress. Triiodothyronine (T3) and tetra iodothyronine (T4) have physiological effects on the cardiovascular system. T3 and T4 decrease peripheral vascular resistance and have a positive chronotropic and inotropic effect. The purpose of our study was to examine the relationship among T3, T4 levels and mortality after orthotopic heart transplantation.

\section{Multivariate Cox regressional analysis for $30-$
postoperative MCS and extended MV (>72h)}

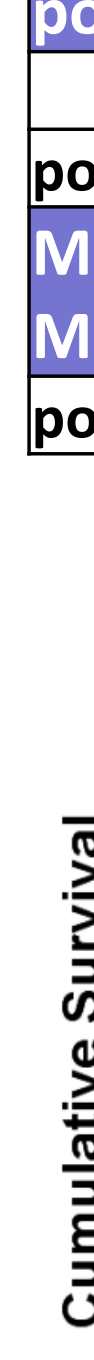

OR 18.26

$95 \% \mathrm{Cl}$

\begin{tabular}{|c|c|} 
& $\mathbf{p}$ \\
\hline $\mathbf{2 7 2 . 2 2}$ & $\mathbf{0 . 0 3 5}$ \\
\hline
\end{tabular}

\begin{tabular}{|l|l|l|l|}
\hline 21.86 & 2.42 & 197.74 & 0.006 \\
\hline
\end{tabular}

Thyroid hormones serum concentration in serious systemic stress

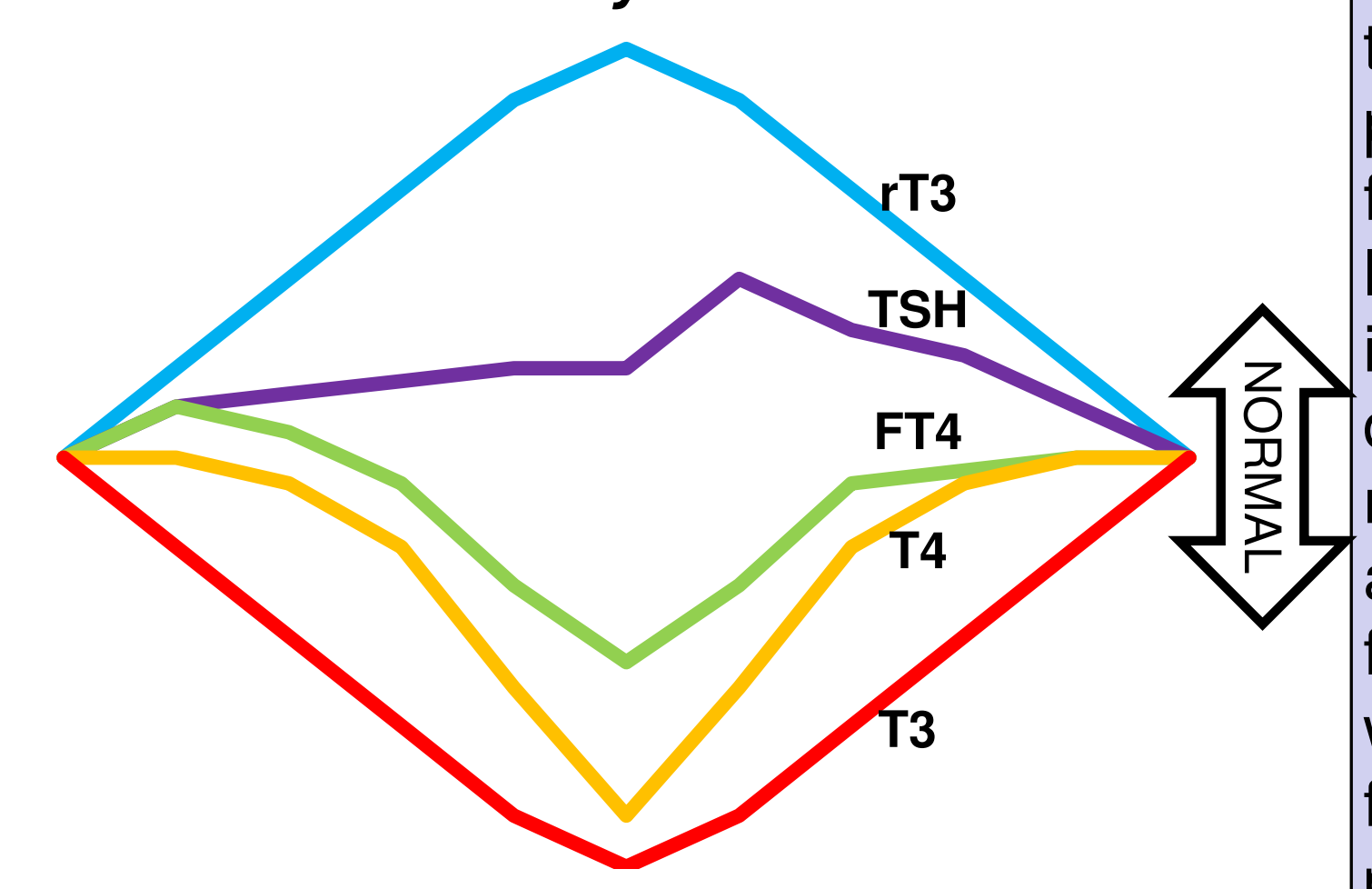

mild $\rightarrow$ moderate $\rightarrow$ severe $\rightarrow$ recovery

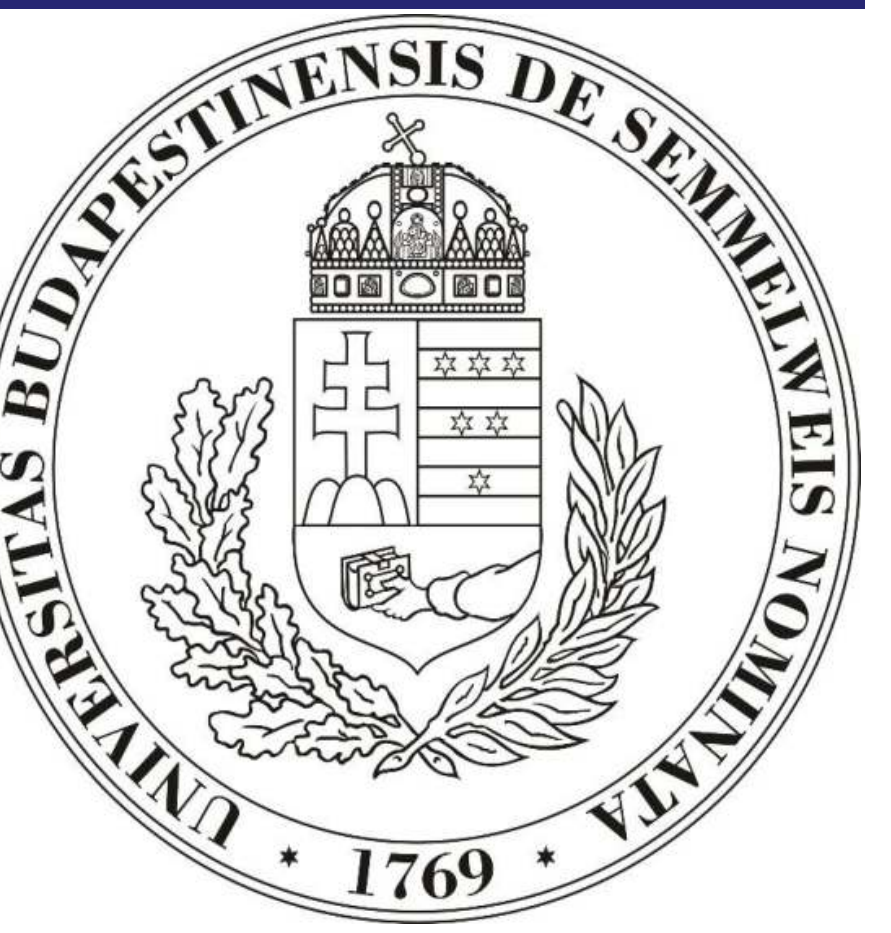

\section{Methods:}

Our retrospective study was approved by the IRB and contained the data of 127 patients undergoing orthotopic heart transplantation. Beside postoperative thyroid function (T3, T4, TSH levels), vasopressorinotropic needs, demographic parameters, mechanical cardiac support and UNOS (United Network for Organ Sharing) score were investigated. Eighty five patients had postoperative thyroid levels. We used Mantel-Cox logrank test and multivariable Cox regression for further investigation.

\section{Results and Conclusions:}

12 of the $85(14.1 \%)$ patients died, the mean survival time was 466 days after transplantation. 30-days mortality was $7.1 \%$. 8 (9.4\%). We have found independent relationship among both lower T3 and T4 and 30-days and total mortality $(p=0.035$ and $p=0.006$ respectively).

Based on the above results, thyroid function should be monitored after heart transplantation. Early thyroid hormone replacement therapy might be discussed.

Klein I: Thyroid hormone and the cardiovascular system. The American journal of medicine. 88:631-637, 1990

Van den Berghe G: Non-thyroidal illness in the ICU: a syndrome with different faces. Thyroid: ICU: a syndrome with different faces. Thyr Association. 24:1456-1465, 2014. 